\title{
EDITORIAL
}

\section{Athlete/doctor relationship - is the knowledge base shifting?}

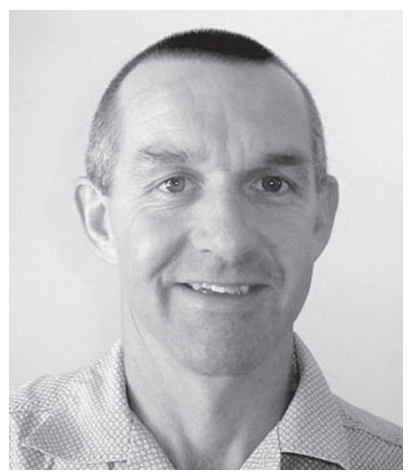

I had a thought-provoking moment while listening to a podcast (cyclingsnews.com, 26 March 2009). David Millar, the 32-year-old 2007 British National cycling road-racing champion, was interviewed about his accident in the last stage of the 2009 Paris-Nice race a few weeks earlier during which he crashed and broke his collarbone. Not that breaking a collarbone was particularly unusual, as this is the second most common injury sustained by cyclists crashing in pelotons - skin lacerations are the most common under such circumstances. What I found unusual was the manner in which Millar discussed the management of the injury. He spoke with confidence about his injury and how it differed from the collarbone injury of Lance Armstrong. He explained how he had consulted at least 10 specialists for an opinion on how to best treat the injury so that he would be ready to ride in the Tour de France starting on 4 July. After obtaining 5 different opinions he settled for the 'tight rope' procedure and recruited a specialist in Sheffield, UK, who had done 50 such operations in 2008. Although this procedure requires 4 weeks of immobilisation, Millar chose this method over the other methods that had a quicker recovery time but poorer long-term prognoses. The point to this detailed explanation is that it heralds a new era in sports medicine where specialist practitioners are held accountable and their records and experience carefully examined before a decision is made about utilising their services. This scenario has intriguing consequences, particularly in team sports where there is usually one doctor who serves the whole team, and a coach who may put subtle, or not so subtle, pressure on the doctor to accelerate the treatment of the injured player. Sooner, rather than later, an incident involving a high-profile injured player will be the catalyst for the credentials of the doctor managing the case to be exposed. It does not take much insight to see that a sharp legal brain will have a field day with this, particularly if the skills of the doctor or other support staff do not meet the highest standards.

On the point of standards, this edition of SAJSM includes the latest consensus on the management of concussion injuries. The document represents the consensus view of scientists and practitioners who attended the meeting in Zurich in November 2008. This is an important document and therefore we have taken the unusual step of publishing it in SAJSM, free of copyright restriction, even though it will also be published in 10 other journals around the world (American Journal of Sports Medicine, Journal of Science and Medicine in Sport, Journal of Athletic Training, Physical Medicine and Rehabilitation, Journal of Clinical Neuroscience, Scandinavian Journal of Science and Medicine in Sport, Physician and Sports Medicine, British Journal of Sports Medicine, Clinical Journal of Sports Medicine, Journal of Clinical Sports Medicine (Japanese journal) and Neurosurgery. As explained in the preamble to the document (page 36), it has been developed for the use of physicians, therapists, certified athletic trainers, health professionals, coaches and others involved in the care of injured athletes, whether at the recreational, elite or professional level. This approach of preparing consensus documents for managing an injury/condition within sports medicine is gathering momentum. Perhaps it is time to do a stock take of all the controversial management practices in sports medicine and systematically prepare consensus documents on management, with input from the best practitioners in the world. This certainly will lower the risk of a disgruntled athlete instigating legal charges against any medical service provider for not using best practice medicine.

Mike Lambert Editor-in-Chief 